\title{
Excesso de peso e variáveis associadas em escolares de Itajaí, Santa Catarina, Brasil
}

\author{
Overweight and associated variables in schoolchildren in Itajaí \\ in the State of Santa Catarina, Brazil
}

Carolina Kneipp ${ }^{1}$

Fernanda Habitzreuter ${ }^{1}$

Tatiana Mezadri ${ }^{1}$

Doroteia Aparecida Höfelmann ${ }^{2}$

${ }^{1}$ Curso de Nutrição, Centro de Ciências da Saúde, Universidade do Vale do Itajaí. R. Uruguai, nº458, Centro. 88302-202 Itajaí SC Brasil.mezadri@univali.br ${ }^{2}$ Departamento de Nutrição, Universidade Federal do Paraná.

\begin{abstract}
The research objective was to evaluate the association between socioeconomic and demographic variables of health-related behaviors and characteristics of the home environment and the prevalence of overweight among students from 1 st to 5 th grade in the city of Itajai in the State of Santa Catarina, Brazil. Socioeconomic and demographic variables were identified through a questionnaire administered to parents/guardians. Nutritional status was determined by body mass index and waist circumference. The association between excess weight and other variables was investigated using Pearson's Chi-Square and the Linear Trend test. The population consisted of 417 children, which represented $95.2 \%$ of the sample. The data suggest that $44 \%$ of children were overweight, which was associated with: a better quality diet $(49.4 \%, p=0.051)$, lower frequency of consumption of snack foods, habit of eating meals while watching the television, overweight parents $(51 \%, p=0,002)$, the score of family nutrition $\left(51.9 \%, p=0.029^{* *}\right)$ and physical activity in children. It is suggested that health actions must consider the family environment and the social context of children to promote healthier lifestyles. Key words Child, Nutritional status, Overweight, Family, Eating habits
\end{abstract}

Resumo Objetivou-se avaliar a associação das variáveis socioeconômicas, demográficas, dos comportamentos relacionados à saúde e características do ambiente familiar com a prevalência de excesso de peso em alunos do $1^{\circ}$ ao $5^{\circ}$ ano de escolas do município de Itajaí, Santa Catarina. As variáveis socioeconômicas, demográficas e referentes aos comportamentos e características do ambiente familiar foram identificadas por meio de questionário aplicado aos pais/responsáveis. Foi aferido e determinado o estado nutricional pelo Índice de Massa Corporal e circunferência da cintura. A associação entre o excesso de peso e as demais variáveis foi investigada por meio do teste do Qui-Quadrado de Pearson e de Tendência Linear. A população constituía-se de 417 escolares, 95,2\% da amostra. Os dados indicaram que 44,0\% apresentaram excesso de peso, que esteve associado à melhor qualidade da dieta $(49,4 \%$, $p=0,051)$, menor frequência de consumo de biscoitos e salgadinhos, hábito de realizar refeições em frente à televisão, ao excesso de peso dos pais ( $51 \%, p=0,002)$, ao escore da atuação da família na alimentação $(51,9 \%, p=0,029)$ e atividade física das crianças. Sugere-se que as ações em saúde devem considerar o entorno familiar e o contexto social das crianças para fomentar estilos de vida mais saudáveis.

Palavras-chave Criança, Estado nutricional, Sobrepeso, Família, Hábitos alimentares 


\section{Introdução}

O Brasil é um país de contrastes, a população de crianças e jovens convive com agravos nutricionais decorrentes, principalmente, de uma alimentação inadequada em termos de qualidade e quantidade. Por estarem em contínuo crescimento e desenvolvimento, as crianças são especialmente susceptíveis a esses agravos, evoluindo com repercussões sistêmicas, atuais e futuras ${ }^{1}$.

Observa-se no Brasil aumento expressivo na prevalência de crianças com excesso de peso. Segundo dados do Instituto Brasileiro de Geografia e Estatística ${ }^{2}$ uma em cada três crianças brasileiras de cinco a nove anos estava acima do peso recomendado pela Organização da Mundial da Saúde. Portanto, o sobrepeso e a obesidade identificamse como problema de saúde pública, emergindo como preocupação de toda a sociedade.

A obesidade é uma questão contemporânea acompanhada de facilidades nas realizações das tarefas diárias observada com o avanço da tecnologia. Possui causa multifatorial que além de resultar em acúmulo excessivo de gordura no organismo, pode estar vinculada ao acometimento de doenças, como hipercolesterolemia, hipertrigliceridemia, hipertensão arterial e diabetes; surgindo na infância e continuando ao longo da vida. Além disso, sua repercussão inclui aspectos sociais importantes ligados à discriminação sofrida por indivíduos com excesso de massa corporal, o que pode levar a menor habilidade social, e a resultados desfavoráveis futuramente no trabalho ${ }^{3-6}$.

A adoção de hábitos alimentares e comportamentais pouco saudáveis, como adesão a um padrão de dieta rica em alimentos com alta densidade energética e baixa concentração de nutrientes, aumento do consumo de alimentos ultraprocessados e ingestão excessiva de nutrientes como sódio, gorduras, açúcar e uma abundância de passatempos sedentários para crianças, o deslocamento passivo e o tempo excessivo em frente da televisão ou computador levaram ao aumento excessivo de peso no Brasil ${ }^{7,8}$.

A formação dos hábitos alimentares acontece de modo gradual, principalmente durante a primeira infância, detendo aos pais o papel de primeiros educadores nutricionais. Valores culturais, sociais, afetivos, emocionais e comportamentais também estão envolvidos neste processo e necessitam ser cuidadosamente associados às propostas de mudanças ${ }^{9,10}$.

O contexto social adquire um papel preponderante no processo de desenvolvimento do comportamento alimentar, principalmente nas estratégias que os pais utilizam para a criança alimentar-se ou para aprender a comer alimentos específicos. Estes comportamentos podem apresentar estímulos tanto adequados quanto inadequados na obtenção das preferências alimentares da criança e no autocontrole da ingestão alimentar ${ }^{11}$.

A família é fundamental na construção do hábito alimentar das crianças, assim, é importante avaliar a influência do estilo de vida dos pais no comportamento delas. Os hábitos de ingestão parental mostraram-se determinantes potenciais no consumo frequente da crianç ${ }^{12}$.

A baixa escolaridade dos pais, em particular a materna, pode contribuir de forma significativa para a carência de alimentos e para uma escolha inadequada dos mesmos, determinando agravos nutricionais nas crianças ${ }^{13}$.

Estudos demonstram associação entre a obesidade infantil e o histórico parental, assim como a influência da família nos hábitos alimentares, acesso à tecnologia e incentivo à atividade físi$\mathrm{ca}^{14,15}$.

Foram identificadas poucas pesquisas sobre a influência familiar no estado nutricional das crianças no Brasil ${ }^{16,17}$.

De acordo com o exposto, o presente estudo teve como objetivo avaliar a associação das variáveis socioeconômicas, demográficas, dos comportamentos relacionados à saúde e características do ambiente familiar com a prevalência de excesso de peso em alunos do $1^{\circ}$ ao $5^{\circ}$ ano de escolas do município de Itajaí, Santa Catarina.

\section{Metodologia}

Trata-se de um estudo transversal com escolares do primeiro ao quinto ano de três escolas da área urbana da rede municipal de Itajaí, Santa Catarina em 2013.

O município estudado localiza-se no Vale do Itajaí, a $91 \mathrm{~km}$ de Florianópolis, capital catarinense. A cidade tem como economia o porto, comércio atacadista de combustível e pesca, além do setor de produção industrial com grande atuação com o comércio de gêneros alimentícios ${ }^{18}$. Itajaí apresentava uma população de 183.388 habitantes ${ }^{19} \mathrm{e}$ um contingente de 25.699 crianças matriculadas no ensino infantil e fundamental ${ }^{20}$. Em julho de 2012, na área urbana, as escolas municipais apresentavam 9.334 alunos matriculados nas séries iniciais, distribuídos em 31 unidades escolares.

As três escolas selecionadas contavam com 919 alunos do $1^{\circ}$ ao $5^{\circ}$ ano. As unidades escolares 
avaliadas foram indicadas pela Secretaria de Educação de Itajaí por não terem participado do Projeto Saúde na Escola, em que os escolares dos municípios participantes são analisados em vários âmbitos da área da saúde, inclusive peso e altura.

Para cálculo da amostra considerou-se prevalência de $50 \%$ do desfecho para maximizar tamanho amostral, com margem de erro de $4 \%$ e nível de confiança de $95 \%$, o que totalizou 365 alunos. Para compensar eventuais recusas e permitir maior poder estatístico nas análises entre desfecho e exposições investigadas acrescentou-se $20 \%$, o que totalizou uma amostra de 438 escolares. As estimativas foram calculadas no programa Epi Info 6.04 (Center of Control of Diseases).

A coleta de dados foi realizada por acadêmicos do curso de Nutrição da Universidade do Vale do Itajaí devidamente treinados e incluiu dados antropométricos das crianças e aplicação de questionário para seu pai/responsável. Na avaliação dos fatores socioeconômicos foram consideradas as seguintes variáveis: renda familiar média per capita em reais categorizada em tercis $\left(1^{\circ}\right.$ Tercil $\mathrm{R} \$$ 83,33 à $R \$ 500,00,2^{\circ}$ Tercil $R$ \$501,00 à R\$750,99 e $3^{\circ}$ Tercil R\$ 751,00 à R\$3.333,33), horas trabalhadas fora de casa, cor da pele (branca ou parda, negra, amarela e indígena) ${ }^{2}$, e escolaridade dos pais (até 8 anos, de 9 a 11 anos e 12 anos ou mais).

Dados adicionais sobre a criança incluíram: ano de estudo ( $1^{\circ}$ ao $5^{\circ}$ ano), realização do café da manhã (sim - diariamente e não - de 0 a 6 dias na semana), alimentação em frente à televisão (não, sim), tipo de transporte que usava para ir à escola, classificado em: transporte ativo (a pé e de bicicleta) ou inativo (de ônibus e de carro), horas diárias em que a criança brincava ativamente.

O consumo alimentar dos escolares foi avaliado por meio de Questionário de Frequência Alimentar (QFA) compreendendo a frequência de 18 itens alimentícios. A qualidade da alimentação foi calculada por meio do Índice Alimentação do Escolar (Ales), segundo proposta de Molina et al..$^{21}$, baseada no Guia Alimentar para a População Brasileira ${ }^{22}$. O Î́ndice de Ales abordou uma pontuação positiva ou negativa sobre os itens alimentícios investigados, segundo a sua frequência de consumo e qualidade ${ }^{21}$. Após computação da pontuação de cada indivíduo, o escore foi analisado conforme as medianas de distribuição: alimentação de pior qualidade (-6 a 4 pontos) e alimentação de melhor qualidade (5 a 13 pontos).

Os comportamentos familiares relacionados à alimentação e à prática de atividade física foram avaliados por meio do questionário Family
Nutrition and Physical Activity (FNPA), em sua versão na língua portuguesa, o qual foi desenvolvido para determinar a força da evidência na relação entre excesso de peso ou obesidade com a atividade física específica e comportamentos relacionados à dieta. O instrumento é constituído por construtos baseados em análises de evidências, em colaboração com a American Dietetic Association. Por meio do FNPA foi realizado um cálculo para a pontuação de cada indivíduo, o que gerou um escore que foi dividido em tercis: baixo ( 0 - 49 pontos), médio ( 50 - 55 pontos) e alto (56 - 74 pontos $)^{23}$. Um total elevado de pontos implica em um ambiente familiar e comportamentos mais favoráveis positivamente contra uma baixa pontuação que significa maior risco para um ambiente familiar e comportamental menos favorável.

Foram aferidos os dados antropométricos de peso, estatura e circunferência da cintura nas crianças, sendo que o peso e altura dos pais/responsáveis foi autorreferido. Para a pesagem foi utilizada uma balança digital da marca SCALA ${ }^{\circledR}$ e para a estatura um estadiômetro CAUMAQ ${ }^{\circledR}$. Em ambas as avaliações os escolares se apresentaram sem adereços no cabelo, descalços e com os braços estendidos ao lado do corpo ${ }^{24}$. A partir dos dados de peso e estatura foi calculado e classificado o Índice de Massa Corporal (IMC) para idade com auxílio do programa ANTHRO plus ${ }^{25}$, utilizando os pontos de corte propostos pela Organização Mundial da Saúde ${ }^{25}$.

A circunferência da cintura foi medida com o auxílio de uma fita antropométrica inextensível no perímetro mais estreito, entre a última costela e a crista ilíaca sem comprimir os tecidos, os valores foram avaliados segundo Taylor et al. ${ }^{26}$.

Os questionários foram revisados e codificados. A dupla-digitação dos dados foi efetuada em planilha eletrônica de forma independente. Posteriormente os registros foram conferidos no programa Epilnfo, e as diferenças nas planilhas ajustadas.

Para descrever as variáveis quantitativas foram calculadas as médias e os desvios-padrão (DP), valores mínimos, medianos e máximos, além dos percentis 25 e 75 quando necessários. As variáveis categóricas foram descritas por meio de suas frequências absolutas (n) e relativas (\%). A associação entre o excesso de peso (sobrepeso e obesidade) e as demais variáveis foi investigada por meio do teste do Qui-Quadrado de Pearson. Adicionalmente as variáveis categóricas ordinais tiveram sua associação com o desfecho testada por meio do teste de Tendência Linear. Asso- 
ciações entre o desfecho e os itens do QFA e do FNPA foram investigadas por meio do teste não paramétrico de Mann Whitney, considerando a distribuição não simétrica destas variáveis. As análises foram realizadas no programa Stata 10.0. $\mathrm{O}$ valor de $\mathrm{p} \leq 0,05$ foi considerado significativo nas análises.

O projeto de pesquisa foi aprovado pelo Comitê de Ética em Pesquisa da Universidade do Vale do Itajaí. Os pais dos participantes deste estudo assinaram o Termo de Consentimento Livre e Esclarecido.

\section{Resultados}

A amostra foi constituída de 438 crianças, com uma perda amostral de 4,8\%, totalizando 417 crianças. Dos pais/responsáveis que responderam ao questionário $(95,2 \%)$, a maioria era do sexo feminino $(84,9 \%)$, com idade média de 36,1 anos (mínimo 21 e máximo 64 anos). Observouse predominância da cor de pele branca $(74,9 \%) \mathrm{e}$ renda per capita de $\mathrm{R} \$ 83,33$ à $\mathrm{R} \$ 500,00$ (45,5\%). Aproximadamente metade dos pais e mães apresentou de 9 a 11 anos de estudo $(49,9 \%$ e 48,2\%, respectivamente) e excesso de peso $(51,3 \%, \mathrm{n}=$ 201) (Tabela 1).

As crianças se distribuíram homogeneamente com relação ao sexo (50,6\% feminino), com média de 8,8 anos (DP 1,5$)$. Mais da metade $(66,8 \%$, $\mathrm{n}=269)$ realizava o café da manhã diariamente e a maioria das crianças consumia as refeições em frente à televisão $64,9 \%(\mathrm{n}=268)$. Um terço das crianças apresentou baixos escores de atuação da família na alimentação e na atividade física (0-49 pontos) (Tabela 1). Os escolares brincavam ativamente 2,9 horas por dia e jogavam videogame e computador por 5,3 horas semanais.

Observou-se que $44,0 \%(\mathrm{n}=182)$ estavam com excesso de peso e $24,7 \%$ com excesso de gordura na região abdominal (Tabela 1). As crianças com excesso de peso apresentaram maior frequência de consumo semanal de carnes, e menor de biscoitos recheados e salgadinhos (Tabela 2).

Os itens do escore de atuação da família na alimentação e na atividade física das crianças associados ao excesso de peso foram: consumo de leite desnatado, atividade física e atividade física com treinador (Tabela 3).

Não foi evidenciada associação estatisticamente significativa entre o excesso de peso e as variáveis: sexo do responsável, renda familiar, escolaridade dos pais, tempo de trabalho, sexo da criança, ano em que estuda, meio de deslo-
Tabela 1. Caracterização da amostra estudada, segundo pais/responsáveis e escolares da rede municipal de ensino - Itajaí, SC, 2013.

\begin{tabular}{|c|c|c|}
\hline Variáveis & $\mathbf{n}$ & $\%$ \\
\hline \multicolumn{3}{|l|}{ Responsável } \\
\hline $\begin{array}{l}\text { Sexo }(\mathrm{n}=410) \\
\text { Masculino } \\
\text { Feminino }\end{array}$ & $\begin{array}{r}62 \\
348\end{array}$ & $\begin{array}{l}15,1 \\
84,9\end{array}$ \\
\hline $\begin{array}{l}\text { Cor da pele }(\mathrm{n}=403) \\
\text { Branca } \\
\text { Não branca }\end{array}$ & $\begin{array}{l}302 \\
101\end{array}$ & $\begin{array}{l}74,9 \\
25,1\end{array}$ \\
\hline $\begin{array}{l}\text { Renda familiar per capita }(\mathrm{n}=319) \\
1^{\circ} \text { tercil }(\mathrm{R} \$ 83,33 \text { à } \mathrm{R} \$ 500,00) \\
2^{\circ} \text { tercil }(\mathrm{R} \$ 501,00 \text { à } \mathrm{R} \$ 750,99) \\
3^{\circ} \text { tercil }(\mathrm{R} \$ 751,00 \text { à } \mathrm{R} \$ 3333,33)\end{array}$ & $\begin{array}{r}145 \\
82 \\
92\end{array}$ & $\begin{array}{l}45,5 \\
25,7 \\
28,8\end{array}$ \\
\hline $\begin{array}{l}\text { Escolaridade do Pai }(\mathrm{n}=417) \\
\text { Até } 8 \text { anos } \\
9 \text { a } 11 \text { anos } \\
12 \text { anos e mais }\end{array}$ & $\begin{array}{r}126 \\
208 \\
83\end{array}$ & $\begin{array}{l}30,2 \\
49,9 \\
19,9\end{array}$ \\
\hline $\begin{array}{l}\text { Escolaridade da Mãe }(\mathrm{n}=417) \\
\text { Até } 8 \text { anos } \\
9 \text { a } 11 \text { anos } \\
12 \text { anos e mais }\end{array}$ & $\begin{array}{l}115 \\
201 \\
101\end{array}$ & $\begin{array}{l}27,6 \\
48,2 \\
24,2\end{array}$ \\
\hline $\begin{array}{l}\text { Tempo de trabalho semanal }(n=336) \\
0 \text { a } 39 \text { horas } \\
40 \text { horas ou mais }\end{array}$ & $\begin{array}{l}159 \\
177\end{array}$ & $\begin{array}{l}47,3 \\
52,7\end{array}$ \\
\hline $\begin{array}{l}\text { Excesso de peso }(\mathrm{n}=392) \\
\text { Não } \\
\text { Sim }\end{array}$ & $\begin{array}{l}191 \\
201\end{array}$ & $\begin{array}{l}48,7 \\
51,3\end{array}$ \\
\hline
\end{tabular}

camento trajeto casa/escola, consumo de café da manhã diariamente. Por outro lado, o excesso de peso dos pais esteve associado ao excesso de peso infantil. Crianças com excesso de peso apresentaram: maior idade, dieta de melhor qualidade, hábito de realizar refeições em frente à televisão, bem como maiores escores de participação familiar na alimentação e na atividade física. Praticamente todas as crianças com obesidade abdominal apresentaram excesso de peso $(98,1 \%)$ (Tabela 4).

\section{Discussão}

Os resultados desta pesquisa indicaram que quase metade das crianças apresentou excesso de peso, o qual esteve associado: à melhor qualidade da dieta, à maior frequência de consumo de carne e menor de biscoitos e salgadinhos, ao hábito de realizar refeições em frente à televisão, 
Tabela 1. continuação

\begin{tabular}{|c|c|c|}
\hline Variáveis & $\mathbf{n}$ & $\%$ \\
\hline \multicolumn{3}{|l|}{ Criança } \\
\hline \multicolumn{3}{|l|}{ Sexo $(n=413)$} \\
\hline Masculino & 204 & 49,4 \\
\hline Feminino & 209 & 50,6 \\
\hline \multicolumn{3}{|l|}{ Idade $(\mathrm{n}=414)$} \\
\hline $1^{\circ}$ Tercil - 6,27 - 8,00 & 138 & 33,3 \\
\hline 2o Tercil - 8,01 - 9,65 & 138 & 33,3 \\
\hline 3o Tercil - 9,66 - 11,64 & 138 & 33.3 \\
\hline \multicolumn{3}{|l|}{ Ano de estudo $(n=415)$} \\
\hline $1^{\circ}$ ano & 83 & 20,0 \\
\hline $2^{\circ}$ ano & 75 & 18,1 \\
\hline $3^{\circ}$ ano & 88 & 21,2 \\
\hline $4^{\circ}$ ano & 80 & 19,3 \\
\hline $5^{\circ}$ ano & 89 & 21,5 \\
\hline \multicolumn{3}{|c|}{$\begin{array}{l}\text { Frequência semanal café da manhã } \\
(\mathrm{n}=403)\end{array}$} \\
\hline $0-6$ dias & 134 & 33,3 \\
\hline Todos os dias & 269 & 66,8 \\
\hline \multicolumn{3}{|c|}{ Refeição em frente à televisão $(\mathrm{n}=413)$} \\
\hline Não & 145 & 35,1 \\
\hline Sim & 268 & 64,9 \\
\hline \multicolumn{3}{|c|}{$\begin{array}{l}\text { Meio de deslocamento, trajeto casa/ } \\
\text { escola }(\mathrm{n}=406)\end{array}$} \\
\hline Inativo & 235 & 57,9 \\
\hline Ativo & 171 & 42,1 \\
\hline \multicolumn{3}{|l|}{ Excesso de peso $(n=414)$} \\
\hline Não & 232 & 56,0 \\
\hline Sim & 182 & 44,0 \\
\hline \multicolumn{3}{|c|}{ Obesidade Abdominal $(\mathrm{n}=414)$} \\
\hline Não & 311 & 74,6 \\
\hline Sim & 104 & 24,7 \\
\hline \multicolumn{3}{|c|}{$\begin{array}{l}\text { Escore de atuação da família na } \\
\text { alimentação e atividade física }\end{array}$} \\
\hline Baixo $(0-49$ pontos $)$ & 153 & 36,7 \\
\hline Médio (50 - 55 pontos) & 127 & 30,5 \\
\hline Alto (56 - 74 pontos) & 137 & 32,9 \\
\hline
\end{tabular}

e ao excesso de peso dos pais. Além disso, o escore da atuação da família na alimentação e atividade física das crianças também foi superior entre aquelas com excesso de peso.

$\mathrm{O}$ excesso de peso tem atingido prevalências elevadas na população infantil nas últimas décadas. Dados da POF 2008-2009 para crianças de 5 a 9 anos de idade, que utilizaram o Índice de Massa Corporal para idade, indicaram prevalência de excesso de peso de $33,4 \%{ }^{2}$. Onis et al. ${ }^{27}$, ao analisarem dados relativos a 144 países, estimaram 43 milhões de crianças com sobrepeso e obesidade no ano de 2010, sendo que, destes, 35 milhões encontravam-se nos países em desenvol- vimento, com uma evolução de 4,2\% em 1990 para $6,7 \%$ em 2010. Em estudo realizado com escolares de 7 a 10 anos em Florianópolis (SC) verificou-se que $34,5 \%$ apresentaram sobrepeso ou obesidade ${ }^{28}$.

$\mathrm{O}$ excesso de peso foi frequente entre as crianças de 8 a 9 anos. Resultado semelhante foi encontrado no estudo de Damasceno et al. ${ }^{29}$, no qual a prevalência para sobrepeso, obesidade e obesidade central esteve mais elevada aos 9 anos, com $19 \%$, 9,5\% e $35,9 \%$, respectivamente. Este fato pode ser explicado pela fase de repleção pré -puberal, que ocorre por volta dos 8 a 10 anos de idade com acúmulo de tecido adiposo como forma de fazer reserva energética para posterior estirão de crescimento e desenvolvimento acentuados que ocorrerão na adolescência ${ }^{30}$.

Na população investigada do presente estudo não se observou associação estatisticamente significativa entre a escolaridade dos pais e o excesso de peso das crianças. Contudo, a relativa homogeneidade socioeconômica dos escolares avaliados, todos provenientes de instituições públicas de ensino, pode dificultar a discriminação dos efeitos da renda e da escolaridade nos desfechos investigados. Além disto, na faixa de renda investigada, a relativa melhor condição socioeconômica pode refletir em maior poder de aquisição de alimentos ricos em gordura saturada, açúcares e sódio ${ }^{31,32}$.

Apesar das desigualdades econômicas e socioculturais da população brasileira, a globalização universalizou o acesso à mídia. As facilidades tecnológicas possibilitaram o acesso de populações socialmente vulneráveis ao universo midiático e às suas influências, transformando as condições materiais de vida, ao modificar os hábitos de consumo nessas populações ${ }^{33}$.

Filhos de pais com excesso de peso apresentaram maior prevalência do desfecho. Resultado similar àquele observado por outros autores ${ }^{34,35} \mathrm{e}$ que destaca o compartilhamento de hábitos alimentares, e relacionados ao estilo de vida entre pais e filhos ${ }^{28,36}$.

Entre as crianças que possuíam o hábito de realizar as refeições em frente à televisão às vezes ou sempre, a prevalência de excesso de peso foi superior. Estudos internacionais com crianças mostram que a televisão aumenta a ingestão de alimentos e a obesidade. Estas investigações demonstraram que ampliações no aumento do percentual de gordura corporal e elevação do diabetes na infância estão fortemente associadas com exposição prolongada à televisão ${ }^{22,37}$. As propagandas divulgadas pelo aparelho também favorecem o desenvolvimento de hábitos alimentares 
Tabela 2. Frequência de consumo alimentar semanal segundo excesso de peso em crianças da rede municipal de ensino - Itajaí, SC, 2013.

\begin{tabular}{|c|c|c|c|}
\hline \multirow[b]{2}{*}{ Alimentos/Bebidas } & \multicolumn{2}{|c|}{ Excesso de peso } & \multirow[b]{2}{*}{$\begin{array}{c}\text { Valor } \\
\text { p }^{*}\end{array}$} \\
\hline & $\begin{array}{c}\text { Não } \\
\text { Med (p25 - p75) }\end{array}$ & $\begin{array}{c}\operatorname{Sim} \\
\operatorname{Med}(\mathrm{p} 25-\mathrm{p} 75)\end{array}$ & \\
\hline Macarrão instantâneo & $0(0-2)$ & $0(0-1)$ & 0,376 \\
\hline Carne/Frango & $5(4-7)$ & $6(4-7)$ & 0,042 \\
\hline Peixes e mariscos & $1(0-2)$ & $1(0-2)$ & 0,098 \\
\hline Batata frita/ & $1(0-3)$ & $1(0-2)$ & 0,169 \\
\hline \multicolumn{4}{|l|}{ Mandioca ou aipim frito/Banana frita } \\
\hline Salada crua & $2(0-5)$ & $3(0-6)$ & 0,060 \\
\hline Batata cozida/Mandioca ou aipim cozido & $2(0-4)$ & $2(1-4)$ & 0,511 \\
\hline Legumes cozidos (menos batata e mandioca/aipim) & $1(0-3)$ & $2(0-4)$ & 0,092 \\
\hline Maionese /manteiga & $2(0-5)$ & $3(0-5)$ & 0,448 \\
\hline Hambúrguer/Cachorro quente & $1(0-3)$ & $1(0-2)$ & 0,173 \\
\hline Leite/Iogurte/Queijos & $6(4-7)$ & $6(4-7)$ & 0,778 \\
\hline Frutas & $4(2-7)$ & $5(2-7)$ & 0,361 \\
\hline Suco de Fruta Natural & $2(0-4)$ & $2(0-4)$ & 0,788 \\
\hline Refrigerante & $2(1-4)$ & $2(1-4)$ & 0,408 \\
\hline Salgados (coxinha, pastel...) & $1(0-2)$ & $1(0-2)$ & 0,456 \\
\hline Doces/balas/sobremesa & $3(1-5)$ & $3(1-5)$ & 0,339 \\
\hline Presunto/Salame/Mortadela linguiça & $3(0-5)$ & $3(2-5)$ & 0,153 \\
\hline Biscoito (chips/recheado) & $4(1-5)$ & $3(0-4)$ & 0,001 \\
\hline Feijão & $5(3-7)$ & $5(3-7)$ & 0,506 \\
\hline
\end{tabular}

* Teste de Mann Whitney; Med = Mediana; p25= Percentil 25; p75 = percentil 75.

Tabela 3. Associação da atuação da família na alimentação e atividade física com o excesso de peso dos alunos da rede municipal de ensino - Itajaí, SC, 2013.

\begin{tabular}{|c|c|c|c|}
\hline \multirow[b]{2}{*}{ Frequência consumo } & \multicolumn{2}{|c|}{ Excesso de peso } & \multirow[b]{2}{*}{$\begin{array}{c}\text { Valor } \\
p^{*}\end{array}$} \\
\hline & $\begin{array}{c}\text { Não } \\
\text { Med (p25 - p75) }\end{array}$ & $\begin{array}{c}\operatorname{Sim} \\
\operatorname{Med}(\mathrm{p} 25-\mathrm{p} 75)\end{array}$ & \\
\hline Realiza o café da manhã & $3(2-4)$ & $3(2-4)$ & 0,340 \\
\hline Realiza as refeições com a família & $3(3-4)$ & $3(3-4)$ & 0,724 \\
\hline Família não come em frente à TV & $3(2-4)$ & $3(2-4)$ & 0,483 \\
\hline Família não consome fast food & $4(3-4)$ & $3(3-4)$ & 0,328 \\
\hline Família não usa micro-ondas e refeições prontas & $3(3-4)$ & $3(3-4)$ & 0,795 \\
\hline Filho come frutas e vegetais & $2(1-3)$ & $2(2-3)$ & 0,093 \\
\hline Filho não bebe refrigerante ou bebidas açucaradas & $3(2-3)$ & $3(2-3)$ & 0,667 \\
\hline Filho bebe leite desnatado & $1(1-2)$ & $1(1-3)$ & 0,031 \\
\hline Família controla consumo de batata frita de pacote, bolachas & $3(2-3)$ & $3(2-3)$ & 0,780 \\
\hline Família não usa doces como prêmio & $4(4-4)$ & $4(4-4)$ & 0,762 \\
\hline Filho passa menos de 2 horas na frente da TV & $2(1-3)$ & $2(1-3)$ & 0,047 \\
\hline Família limita o tempo de assistir TV & $2(1-3)$ & $2(1-3)$ & 0,128 \\
\hline Família não permite que o filho veja TV no quarto & $3(2-4)$ & $3(2-4)$ & 0,593 \\
\hline Família propicia atividade física & $2(1-3)$ & $2(2-3)$ & $<0,001$ \\
\hline Família incentiva o filho a ser ativo & $3(2-3)$ & $3(2-4)$ & 0,076 \\
\hline Família se exercita junta & $2(1-3)$ & $2(1-3)$ & 0,447 \\
\hline Filho é fisicamente ativo & $3(2-4)$ & $3(2-4)$ & 0,743 \\
\hline Filho participa de atividade com treinador & $1(1-2)$ & $1(1-3)$ & 0,018 \\
\hline Família possui horário pra dormir & $3(2-4)$ & $3(2-4)$ & 0,927 \\
\hline Filho dorme mais de $9 \mathrm{~h}$ por noite & $3(3-4)$ & $3(3-4)$ & 0,713 \\
\hline
\end{tabular}

* Teste do Qui-Quadrado de Pearson. 
Tabela 4. Associação de variáveis com o excesso de peso de crianças da rede municipal de ensino - Itajaí, SC, 2013.

\begin{tabular}{|c|c|c|c|}
\hline \multirow[b]{2}{*}{ Variáveis } & \multicolumn{2}{|c|}{ Excesso de peso } & \multirow[b]{2}{*}{ Valor de $\mathbf{p}^{*}$} \\
\hline & $\begin{array}{l}\text { Não } \\
\text { (n) \% }\end{array}$ & $\begin{array}{l}\text { Sim } \\
(\mathbf{n}) \%\end{array}$ & \\
\hline \multicolumn{4}{|l|}{ Responsável } \\
\hline Sexo $(n=407)$ & & & 0,806 \\
\hline Masculino & (34) 54,8 & (28) 45,2 & \\
\hline Feminino & (195) 56,5 & (150) 43,5 & \\
\hline Cor de pele $(\mathrm{n}=400)$ & & & 0,781 \\
\hline Branco & (169) 56,2 & (132) 43,9 & \\
\hline Parda, Preto, Amarelo ou Indígena & (54) 54,6 & (45) 45,5 & \\
\hline Renda familiar per capita $(\mathrm{n}=316)$ & & & 0,704 \\
\hline $1^{\circ}$ tercil $(\mathrm{R} \$ 83,33$ à $\mathrm{R} \$ 500,00)$ & (77) 54,2 & (65) 45,8 & $0,415^{* *}$ \\
\hline $2^{\circ}$ tercil $(\mathrm{R} \$ 501,00$ à $\mathrm{R} \$ 750,99)$ & (46) 56,1 & (36) 43,9 & \\
\hline 30 tercil $(\mathrm{R} \$ 751,00$ à $\mathrm{R} \$ 3333,33)$ & (55) 59,8 & (37) 40,2 & \\
\hline Escolaridade pai $(\mathrm{n}=414)$ & & & 0,827 \\
\hline Até 8 anos & (69) 55,2 & (56) 44,8 & $0,415^{* *}$ \\
\hline 9 a 11 anos & (114) 55,3 & (92) 44,7 & \\
\hline 12 anos e mais & (49) 59,0 & (34) 41,0 & \\
\hline Escolaridade mãe $(\mathrm{n}=414)$ & & & 0,781 \\
\hline Até 8 anos & (67) 58,8 & (47) 41,2 & $0,515^{* *}$ \\
\hline 9 a 11 anos & (110) 55,3 & (89) 44,7 & \\
\hline 12 anos e mais & (55) 54,5 & (46) 45,5 & \\
\hline Tempo de trabalho semanal $(\mathrm{n}=336)$ & & & 0,663 \\
\hline 0 a 39 horas & (90) 56,6 & (69) 43,4 & \\
\hline 40 horas ou mais & (96) 54,2 & (81) 45,8 & \\
\hline Excesso de peso $(\mathrm{n}=389)$ & & & 0,002 \\
\hline Não & (124) 64,2 & (67) 35,1 & \\
\hline Sim & (97) 49,0 & (168) 51,0 & \\
\hline
\end{tabular}

menos saudáveis, e a redução do tempo dedicado à atividade física ${ }^{33,38}$.

Os resultados deste estudo revelaram a existência de associação entre a circunferência da cintura e o excesso de peso das crianças. Damasceno et al. ${ }^{29}$ ao avaliarem escolares de 6 a 11 anos em Fortaleza (CE), evidenciaram uma forte correlação e associação entre o IMC e a CC, e em $27,2 \%$ da amostra a obesidade central esteve presente. Outros estudos também apresentaram correlação entre o sobrepeso e a circunferência da cintura ${ }^{39,40}$.

Assim como em adultos, as crianças podem sofrer efeitos decorrentes da chamada síndrome plurimetabólica (diabetes mellitus, resistência à insulina, hipertensão arterial sistêmica, dislipidemia, obesidade visceral e albuminúria) quando apresentarem distribuição de gordura do tipo abdominal, sendo que essa ocorrência na infância poderá provocar efeitos negativos e sérios na vida adulta ${ }^{41,42}$. Do ponto de vista clínico, a vigi- lância e a detecção precoce dos fatores de risco associados às dislipidemias são primordiais nas ações preventivas, com destaque para o excesso de peso em crianças, cujo controle por meio da obtenção e manutenção do peso adequado para altura, sexo e idade é factível em ações primárias de atenção à saúde ${ }^{43}$.

Crianças com excesso de peso apresentaram dieta de melhor qualidade do que aquelas com baixo peso ou eutróficas. Bertin et al. ${ }^{44}$, ao avaliarem crianças de 8 a 10 anos de Indaial (SC), encontraram associação significativa entre os bons hábitos alimentares e a obesidade. Porém, no estudo de Triches e Giugliani ${ }^{45}$, ao investigar a associação da obesidade com as práticas alimentares e conhecimentos de nutrição em escolas públicas de duas cidades do Rio Grande do Sul, com crianças de oito a 10 anos, encontraram que a obesidade mostrou-se associada com menos conhecimento de nutrição e práticas alimentares menos saudáveis. 
Tabela 4. continuação

\begin{tabular}{|c|c|c|c|}
\hline \multirow[b]{2}{*}{ Variáveis } & \multicolumn{2}{|c|}{ Excesso de peso } & \multirow[b]{2}{*}{ Valor de $\mathrm{p}^{*}$} \\
\hline & $\begin{array}{l}\text { Não } \\
\text { (n) \% }\end{array}$ & $\begin{array}{l}\text { Sim } \\
\text { (n) } \%\end{array}$ & \\
\hline \multicolumn{4}{|l|}{ Criança } \\
\hline $\operatorname{Sexo}(n=410)$ & & & 0,390 \\
\hline Masculino & (109) 54,0 & (93) 46,0 & \\
\hline Feminino & (121) 58,2 & (87) 41,8 & \\
\hline Idade $(\mathrm{n}=414)$ & & & 0,002 \\
\hline $1^{\circ}$ Tercil - 6,27 - 8,00 & (92) 66,7 & (46) 33,3 & $0,069^{* *}$ \\
\hline $2^{\circ}$ Tercil - 8,01 - 9,65 & (63) 45,7 & (75) 54,3 & \\
\hline 3o Tercil - 9,66 - 11,64 & (77) 55,8 & (61) 44,2 & \\
\hline Ano em que estuda $(\mathrm{n}=412)$ & & & 0,103 \\
\hline $1^{\circ}$ ano & (52) 63,4 & (30) 36,6 & $0,067^{* *}$ \\
\hline $2^{\circ}$ ano & (48) 64,9 & (26) 35,1 & \\
\hline $3^{\circ}$ ano & (42) 47,7 & (46) 52,3 & \\
\hline $4^{\circ}$ ano & (40) 50,6 & (39) 49,4 & \\
\hline $5^{\circ}$ ano & (48) 53,9 & (41) 46,1 & \\
\hline Frequência semanal café da manhã $(\mathrm{n}=403)$ & & & 0,096 \\
\hline $0-6$ dias & (67) 50,0 & (67) 50,0 & \\
\hline Todos os dias & (158) 58,7 & (111) 41,3 & \\
\hline Refeição em frente à TV $(\mathrm{n}=413)$ & & & 0,039 \\
\hline Não & (90) 62,5 & (54) 37,5 & \\
\hline Sim & (138) 51,9 & (128) 48,1 & \\
\hline Meio de deslocamento trajeto casa/escola $(n=406)$ & & & 0,421 \\
\hline Inativo & (128) 54,5 & (107) 45,5 & \\
\hline Ativo & (100) 58,5 & (71) 41,5 & \\
\hline Obesidade Abdominal $(\mathrm{n}=414)$ & & & $<0,001$ \\
\hline Não & (230) 74,0 & (81) 26,0 & \\
\hline Sim & (2) 1,9 & (101) 98,1 & \\
\hline Qualidade da dieta $(\mathrm{n}=414)$ & & & 0,051 \\
\hline Alimentação de pior qualidade & $(142) 60,2$ & (94) 39,8 & \\
\hline Alimentação de melhor qualidade & (90) 50,6 & (88) 49,4 & \\
\hline $\begin{array}{l}\text { Escore de atuação da família na alimentação } \\
\text { e atividade física }(\mathrm{n}=414)\end{array}$ & & & 0,071 \\
\hline Baixo (0 - 49 pontos) & (93) 61,2 & (59) 38,8 & $0,029^{* *}$ \\
\hline Médio (50 - 55 pontos) & (74) 58,3 & (53) 41,7 & \\
\hline Alto (56 - 74 pontos) & (65) 48,2 & (70) 51,9 & \\
\hline
\end{tabular}

“Teste do Qui-Quadrado de Pearson; ** Teste de Tendência Linear.

Sugere-se que a boa qualidade da dieta dos escolares possa ser decorrente do maior interesse em se alimentarem com produtos mais saudáveis e por estarem mais suscetíveis a receberem informações sobre este tema devido à sua condição de obesidade, conforme demonstrado em estudo semelhante ${ }^{45}$.

Dentre os hábitos para redução do peso entre as crianças que o tem em excesso, observou-se maior frequência de consumo de leite desnatado, menor consumo de biscoitos chips e recheados; o que pode indicar maior atenção dos pais com relação a esse problema nos filhos, por meio da adoção de hábitos para tentar reduzir o desfecho entre eles.

O melhor consumo pode também estar parcialmente relacionado a modificações instituídas pelo Governo do Estado, no sentido de melhorar a qualidade da alimentação das crianças como, por exemplo, o Programa Saúde na Escola, criado em 2007, que oferece atenção integral de prevenção, promoção e atenção à saúde de crianças, adolescentes e jovens do ensino básico público. E procurando disciplinar a venda de alimentos 
nas cantinas localizadas dentro das escolas, tanto públicas quanto particulares, alguns governos estaduais, municipais e distritais regulamentaram, via leis ou portarias, a venda de produtos considerados não adequados para o consumo, sobretudo diminuindo o acesso à alimentação inadequada e favorecendo escolhas alimentares mais saudáveis, buscando proteger, assim, a saúde dos estudantes ${ }^{46,47}$.

Neste sentido, hábitos não saudáveis são baseados em um reduzido consumo de frutas, hortaliças e leite e, em contrapartida, em um aumento da ingestão de refrigerantes, doces e salgadinhos fritos, os quais são relacionados às elevadas quantidades de gordura saturada, açúcares e sódio. Isso contribui para o aumento na prevalência de diversas doenças crônicas nessa população, tais como o diabetes, hipertensão e obesidade ${ }^{48-50}$.

O consumo de carnes por parte das crianças com excesso de peso estudadas nesta pesquisa foi maior comparado com as crianças eutróficas. Estudo de Spinelli et al. ${ }^{51}$ encontrou consumo inadequado de porções de carne/ovos em crianças menores de dez anos em São Paulo (SP), que quando associadas ao IMC não foi estatisticamente significativo, diferindo do presente estudo.

As crianças com excesso de peso tiveram maiores escores de participação familiar na alimentação e na atividade física, o que pode ser identificado também pela maior prática de atividade física e atividade física com treinador por essas crianças. Em estudo realizado por Yee et al. ${ }^{52}$ com crianças de 10 anos dos Estados Unidos da América, foram encontrados resultados diferentes do presente estudo, houve associação significativa entre o IMC classificado como sobrepeso e obesidade e o baixo escore total do FNPA.

$O$ efeito significativo para o FNPA indica que esta ferramenta de triagem simples tem validade preditiva para identificar crianças que possam estar em risco de excesso de peso ou obesos. Os resultados demonstram que fatores ambientais modificáveis podem explicar a variância única após a contabilização baseado no IMC da crian$\mathrm{ça}^{53}$.

No estudo de Lopes et al. ${ }^{54}$ as crianças com sobrepeso e obesas praticaram menos atividade física na escola do que os eutróficos. Por outro lado, faziam mais atividade física fora da escola, esta diferença foi estatisticamente significante.

Trost et al. ${ }^{55}$ relataram que os pais atuam como influenciadores diretos e indiretos na ob- tenção de práticas saudáveis dos filhos, principalmente quando diz respeito ao apoio motivacional frente à prática de atividade física. $\mathrm{O}$ suporte social de pais, irmãos e pares é fundamental na adoção de hábitos mais ativos além da questão socioeconômica, traduzida na oportunidade de práticas em diferentes espaços ${ }^{56}$.

Algumas limitações metodológicas devem ser consideradas. Uma delas reside no delineamento do estudo, o qual não permite estabelecer relação de causalidade entre as variáveis estudadas. Desta forma, associações que parecem contraditórias, como a maior participação da família na alimentação e na atividade física, a maior frequência de consumo de leite desnatado, menor de biscoitos, e melhor qualidade da dieta entre as crianças com excesso de peso, podem refletir adoção de comportamentos para reduzir o sobrepeso e a obesidade entre as crianças, bem como respostas dos pais em relação ao comportamento que seria considerado socialmente desejável. Além disto, o questionário de frequência de consumo empregado para avaliar a qualidade da dieta pode não refletir a quantidade de alimentos consumidos em cada dia pelas crianças.

\section{Considerações finais}

A partir dos resultados, conclui-se que $44 \%$ dos escolares apresentaram excesso de peso, o qual esteve associado com as variáveis de melhor qualidade da dieta, hábito de realizar refeições em frente à televisão, excesso de peso dos pais, maior escore de atuação da família na alimentação e atividade física das crianças.

Desta forma, é necessária uma maior atenção das políticas públicas de proteção à criança, principalmente àquelas voltadas para ações de promoção da saúde, educação e monitoramento nutricional, bem como uma maior oferta e facilidades no consumo de alimentos saudáveis como frutas e hortaliças e acesso a ambientes seguros para a prática desportiva e lazer, na tentativa de se atenuar os riscos de obesidade na vida adulta e de doenças decorrentes.

A interação e a integração dos pais e/ou responsáveis é fundamental para uma melhor compreensão do estado nutricional das crianças e dos problemas de saúde relacionados ao sobrepeso e obesidade, o que pode favorecer o reconhecimento e a adoção de estilos de vida mais saudáveis. 


\section{Colaboradores}

C Kneipp e F Habitzreuter participaram da concepção do estudo, coleta de dados e redação do artigo. T Mezadri participou da redação do artigo, revisão crítica do conteúdo e aprovação da versão final. DA Höfelmman participou da concepção do estudo, análise de dados e revisão crítica do conteúdo.

\section{Referências}

1. Goulart LMHF, Viana MRAL. Saúde da criança e do adolescente: agravos nutricionais. Belo Horizonte: Coopmed; 2008.

2. Instituto Brasileiro de Geografia e Estatística (IBGE). Pesquisa de orçamentos familiares 2008-2009: Antropometria e estado nutricional de crianças, adolescentes e adultos no Brasil. Rio de Janeiro: IBGE; 2010.

3. Lorentzen V, Dyeremose V, Larsen BH. Severely overweight children and dietary changes--a family perspective. J Adv Nurs 2012; 68(4):878-887.

4. Palermo TM, Dowd JB. Childhood obesity and human capital accumulation. Soc Sci Med 2012; 75(11):19891998.

5. Pimenta TAM, Rocha R. A obesidade infantil no Brasil: um estudo comparativo entre a PNSN/1989 e a POF/2008-09 entre crianças de 5 a 9 anos de idade. Federation Internationale d'Education Physique 2012; 82(1):66-69.

6. Visram S, Hall TD, Geddes L. Getting the balance right: qualitative evaluation of a holistic weight management intervention to address childhood obesity. J Public Health 2013; 35(2):246-254.

7. Dollman J, Norton K, Norton L. Evidence for secular trends in children's physical activity behaviour. $\mathrm{Br} J$ Sports Med 2005; 39(12):892-897.

8. Brasil. Ministério da Saúde (MS). Política Nacional de Alimentação e Nutrição. Brasília: MS; 2012.

9. Brasil. Ministério da Saúde (MS). Secretaria de Atenção à Saúde. Departamento de Atenção Básica. Guia alimentar para a população brasileira: promovendo a alimentação saudável. Brasília: MS; 2008.

10. Costa MGFA, Nunes MMJC, Duarte JC, Pereira AMS. Conhecimento dos pais sobre alimentação: construção e validação de um questionário de alimentação infantil. Rev. Enf. [periódico na Internet]. 2012 Mar. [acessado 2013 Nov 19]; serIII(6):55-68. Disponível em: http:// www.scielo.gpeari.mctes.pt/scielo.php?script=sci_arttext\&pid=S0874-02832012000100006\&lng=pt. http:// dx.doi.org/10.12707/RIII1158.

11. Ramos M, Stein LM. Desenvolvimento do comportamento alimentar infantil. J Pediatr 2000; 76(Supl. 3):S229-S237.

12. Hennessy E, Hughes SO, Goldberg JP, Hyatt RR, Economos CD. Permissive Parental Feeding Behavior Is Associated with an Increase in Intake of Low-Nutrient-Dense Foods among American Children Living in Rural Communities. J Acad Nutr Diet 2012; 112(1):142-148.

13. Silveira KB, Alves JF, Ferreira HS, Sawaya AL, Florêncio TM. Association between malnutrition in children living in slums, maternal nutritional status, and environmental factors. J Pediatr 2010; 86(3):215-220.

14. Anderson SE, Must A, Curtin C, Bandini LG. Meals in our household: Reliability and initial validation of a questionnaire to assess child mealtime behaviors and family mealtime environments. J Acad Nutr Diet 2012; 112(2):276-284.

15. Baygi F, Dorosty AR, Kelishadi R, Qorbani M, Asayesh $\mathrm{H}$, Mansourian M, Mirkarimi K. Determinants of Childhood Obesity in Representative Sample of Children in North East of Iran. Cholesterol 2012; 2012:875163. 
16. Wilhelm FAX, Antunes JHCL, Schirmer KF. Obesidade infantil e a família: educadores emocionais e nutricionais dos filhos. Psicologia argumento 2007; 25(49):143154.

17. Rossi A, Moreira EAM, Rauen MS. Determinantes do Comportamento Alimentar: Uma Revisão com Enfoque na Família. Rev. Nutr. 2008; 21(6):739-748.

18. Centro de Informática e Automação de Santa Catarina (CIASC). [internet]. Itajaí. 2002. [2013 nov 19]. Disponível em: http://www.sc.gov.br/portalturismo/Default. asp? CodMunicipio $=60 \& \mathrm{Pag}=1$

19. Instituto Brasileiro de Geografia e Estatística (IBGE). Pesquisa de orçamentos familiares 2008-2009: Antropometria e estado nutricional de crianças, adolescentes e adultos no Brasil. Rio de Janeiro: IBGE; 2010.

20. Secretaria de Educação de Itajaí [internet]. Educação em números. 2012. [2013 nov 19]. Disponível em: http://www.educacao.itajai.sc.gov.br/index.php/Artigos-do-Menu-Principal/educacao-em-numeros.pmi

21. Molina MCB, Lopéz PM, Faria CP, NV, Zandonade E. Preditores socioeconômicos da qualidade da alimentação de crianças. Rev Saude Publica 2010; 44(5):785-792.

22. Brasil. Ministério da Saúde (MS). Guia alimentar para a população brasileira: promovendo a alimentação saudável. Brasília: MS; 2005.

23. Ihmels MA, Welk GJ, Eisenmann JC, Nusser SM. Development and preliminary validation of a Family $\mathrm{Nu}$ trition and Physical Activity (FNPA) screening tool. Int J Behav Nutr Phys Act 2009; 6:14.

24. Lohman TG. Applicability of body composition techniques and constants for children and youth. Exerc Sport Sci Rev 1986; 14:325-357.

25. World Health Organization (WHO). Child growth standards: Length/height-for-age, weight-for-age, weight -for-length, weight-for-height and body mass index-forage. Methods and development. Geneva: WHO; 2006. (nonserial publication).

26. Taylor RW, Jones IE, Williams SM, Goulding A. Evaluation of waist circumference, waist-tohip ratio, and the conicity index as screening tools for high trunk fat mass, as measured by dualenergy X-ray absorptiometry, in children aged 3-19 y. Am J Clin Nutr 2000; 72(2):490-495.

27. Onis M, Blossner M, Borghi E. Global prevalence and trends of overweight and obesity among preschool children. Am J Clin Nutr 2010; 92(5):1257-1264.

28. Bernardo CO, Pudla KJ, Longo GZ, Vasconcelos FAG. Fatores associados ao estado nutricional de escolares de 7 a 10 anos: aspectos sociodemográficos, de consumo alimentar e estado nutricional dos pais. Rev Bras de Epidemiol 2012; 15(3):651-661.

29. Damasceno MMC, Fragoso LVC, Lima AKG3, Lima ACS, Viana PCS. Correlação entre índice de massa corporal e circunferência da cintura em crianças. Acta Paul Enferm 2010; 23(5):652-657.

30. Vitolo MR. Nutrição: da gestação ao envelhecimento. Rio de Janeiro: Editora Rubio; 2008.

31. Instituto Brasileiro de Geografia e Estatística (IBGE). Pesquisa de orçamentos familiares 2008-2009: análise do consumo alimentar pessoal no Brasil. Rio de Janeiro: IBGE; 2011.
32. Schuch I, Castro TG, Vasconcelos FAG, Dutra CLC, Goldani MZ. Excesso de peso em crianças de pré-escolas: prevalência e fatores associados. J Pediatr 2013; 89(2):179-188.

33. Motta-gallo S, Gallo P, Cuenca A. Influência da televisão nos hábitos alimentares de crianças do nordeste brasileiro. J Hum Growth Dev 2013; 23(1):87-93.

34. Giugliano R, Carneiro EC. Fatores associados à obesidade em escolares. J Pediatr 2004; 80(1):17-22.

35. Guimarães LV, Barros MBA, Martins MSAS, Duarte EC. Fatores associados ao sobrepeso em escolares. Rev. Nutr. 2006; 19(1):5-17.

36. Pedroni JL, Rech RR, Halpern R, Marin S, Roth LR, Sirtoli M, Cavalli A. Prevalência de obesidade abdominal e excesso de gordura em escolares de uma cidade serrana no sul do Brasil. Cien Saude Colet 2013; 18(5):14171425.

37. Kameswararao AA, Bachu A. Survey of child diabetes and the impact of school level educational interventions in rural schools in Karimnagar district. Int $J$ Diabetes Dev Ctries 2009; 29(2):69-73.

38. Rossi CE, Albernaz DO, Vasconcelos FAG, Assis MA, Pietro, PFD Influência da televisão no consumo alimentar e na obesidade em crianças e adolescentes: uma revisão sistemática. Rev. Nutr. 2010; 23(4):607-620.

39. Soar C, Vasconcelos FAG, Assis MAA. A relação cintura quadril e o perímetro da cintura associados ao índice de massa corporal em estudo com escolares. Cad Saude Publica 2004; 20(6):1609-1616.

40. Ricardo GD, Caldeira GV, Corso ACT. Prevalência de sobrepeso e obesidade e indicadores de adiposidade central em escolares de Santa Catarina, Brasil. Rev Bras Epidemiol 2009; 12(3):424-435.

41. Ferreira JS, Aydos RD. Prevalência de hipertensão arterial em crianças e adolescentes obesos. Cien Saude Colet 2010; 15(1):97-104.

42. Pakpour AH, Yemaninejad MS, Chen HAA. Percepção das mães sobre a obesidade em escolares: uma perspectiva e o impacto de uma intervenção educativa. $J$. Pediatr. 2011; 87(2):169-174.

43. Freitas AEF, Lamounier JA, DD, Oliveira TH, Lacerda DR, Andrade JB, Komatsuzaki R, Freitas VG, Botion LM, Capanema FD. Adiposidade e perfil metabólico em crianças de escolas da zona urbana de Ouro Preto MG. Rev Med Minas Gerais 2013; 23(1):5-12.

44. Bertin RL, Malkowski J, Zutter LCI, Ulbrich AZ. Estado nutricional, hábitos alimentares e conhecimentos de nutrição em escolares. Rev. Paul. de Pediatr. 2010; 28(3):303-308.

45. Triches RM, Giugliani ERJ. Obesidade, práticas alimentares e conhecimento de nutrição em escolares. Rev Saude Publica 2005; 39(4):541-547.

46. Santa Catarina. Lei n 12.061 , de 18 de dezembro de 2001. Dispõe sobre critérios de concessão de serviços de lanches e bebidas nas unidades educacionais, localizadas no Estado de Santa Catarina. Diário Oficial do Estado 2001; 20 dez.

47. Instituto Brasileiro de Geografia e Estatística (IBGE). Pesquisa Nacional da Saúde do Escolar 2012. Rio de Janeiro: IBGE; 2013. 
48. Araújo EDS, Blank N, Oliveira WF. Atividade física e hábitos alimentares de adolescentes de três escolas públicas de Florianópolis/SC. Rev Bras Cineantropom Desempenho Hum 2009; 11(4):428-434.

49. Neutzling MB, Assunção MCF, Malcon MC, Hallal PC, Menezes AMB. Hábitos alimentares de escolares adolescentes de Pelotas, Brasil. Rev. Nutr. 2010; 23(3):379388.

50. Torres P, Linares J, Bonzi N, Giuliano ME, Sanziani L, Pezzotto SM, Luna MB. Prevalência de sobrepeso, obesidad, e hipertensión arterial en adolescentes de uma escuela de arte. Rev Bras Cineantropom Desempenho Hum 2011; 13(4):272-278.

51. Spinelli MGN, Morimoto JM, Freitas APG, Barros CM, Dias DHS, Pioltine MB, Gonçalves PPO, Navarro RB. Estado nutricional e consumo alimentar de pré-escolares e escolares de escola privada. Cien Saude Colet 2013; 6(2):94-101.

52. Yee KE, Eisenmann JC, Carlson JJ, Pfeiffer KA. Association between The Family Nutrition and Physical Activity Screening Tool and cardiovascular disease risk factors in 10-year old children. Int J Pediatr Obes 201; 6(3-4):314-320.

53. Ihmels MA, Welk GJ, Eisenmann JC, Nusser SM, Myers EF. Prediction of BMI Change in Young Children with the Family Nutrition and Physical Activity (FNPA) Screening Tool. Ann Behav Med 2009; 38(1):60-68.

54. Lopes PCS, Prado SRLA, Colombo P. Fatores de risco associados à obesidade e sobrepeso em crianças em idade escolar. Rev Bras Enferm 2010; 63(1):73-78.

55. Trost SG, Sallis JF, Pate RR, Freedson PS, Taylor WC, Dowda M. Evaluating a model of parental influence on youth physical activity. Am J Prev Med 2003; 25(4):277-282.

56. Seabra AF, Mendonça DM, Thomis MA, Anjos LA, Maia JA. Biological and socio-cultural determinants of physical activity in adolescents. Cad Saude Publica 2008; 24(4):721-736.

Artigo apresentado em 29/06/2014

Aprovado em 04/02/2015

Versão final apresentada em 06/02/2015 